\title{
Spatial dependence estimation using FFT of biased
}

\section{covariances}

\author{
Rubén Fernández-Casal ${ }^{\dagger}$ \\ Dept. of Mathematics \\ University of A Coruña
}

\author{
Rosa M. Crujeiras ${ }^{\ddagger}$ \\ Dept. of Statistics and Operations Research \\ University of Santiago de Compostela
}

\begin{abstract}
One of the main problems in geostatistics is the fitting of a valid variogram or covariogram model in order to describe the dependence structure present in the data. The dependence between observations can be also modeled in the spectral domain, but the traditional methods based on the periodogram as an estimator of the spectral density are less suitable for the spatial case. In this work, we propose an estimation method for the covariogram parameters based on the Fast Fourier Transform (FFT) of biased covariances. The performance of this estimator for finite samples is compared through simulation with other classical methods stated in spatial domain, such as weighted least squares and maximum likelihood, as well as with other spectral estimators. Additionally, an example of application to real data is given.
\end{abstract}

Keywords: spatial dependence; geostatistics; covariogram; spectral density; periodogram.

\footnotetext{
${ }^{\dagger}$ Department of Mathematics. Faculty of Computer Science, University of A Coruña. 15071. A Coruña. Spain. E-mail: rfcasal@udc.es.

${ }^{\ddagger}$ Department of Statistics and Operations Research. Faculty of Mathematics, University of Santiago de Compostela. 15782. Santiago de Compostela. Spain. E-mail: emphrcrujeir@usc.es.
} 


\section{Introduction}

Spatial dependence modeling in geostatistics is usually studied through the variogram or covariogram functions, choosing a valid parametric family and applying a fitting criterion in order to obtain the parameter estimates (see e.g. Cressie, 1993, section 2.6). One of the most widely used methods for estimating a variogram consists in fitting a valid parametric model to a set of pilot estimations of the variogram, by Weighted Least Squares (WLS). One of the main advantages of this method is that it is not necessary to assume any distribution on the data. In addition, there are several simulation studies that confirm its good performance (e.g. Zimmerman and Zimmerman, 1991). However, this method has been severely criticized, mainly because the dependence between the pilot estimates is not taken into account in the fitting algorithm (e.g. Stein, 1999, section 6.9). An alternative to this procedure is the use of maximum likelihood (ML) methods. Assuming a Gaussian distribution, ML parameter estimations are obtained maximizing the corresponding likelihood function. Nevertheless, in practice, the variance and dependence parameter estimates may present a non-negligible bias, especially in presence of a non-constant trend. A modification of the ML method that avoids the effect of the trend in the parameter estimation is the Restricted Maximum Likelihood (REML) approach. The idea of this method consists of applying a linear filter to the data such that the distribution of the result (generalized increments) does no longer depend on the trend parameters. Apart from the advantages of ML estimation, these methods allow for joint dependence and trend estimation. The main drawback is the normality assumption, which is not-easy to check and sometimes unsuitable condition. In addition, from a computational point of view, these methods are time consuming for large datasets. Another inconvenience is the possible multimodality of the likelihood surface (e.g. Mardia and Watkins, 1989), which could be overcome using genetic optimization algorithms (see e.g. Goldberg, 1989). For all these reasons, ML estimation methods have been also questioned in the geostatistical literature (e.g. Ripley, 1988).

Spectral methods provide a collection of useful tools for avoiding some of the problems of the spatial domain estimators. Spectral techniques have been 
widely used in time series (see e.g. Brillinger, 1981) and, more recently, for spatially dependent data analysis (Fuentes, 2002; Crujeiras et al., 2007). From the spectral domain, the new target function is the spectral density (the Fourier Transform of the covariance function). In this context, the spatial periodogram is a nonparametric estimator of the spectral density and, although it is asymptotically unbiased, it does not present good consistency properties. Nevertheless, the periodogram has been used as a pilot for adjusting parametric models, for instance, in the Whittle estimation method (Whittle, 1954) for discrete processes. Since periodogram values for different frequencies are asymptotically uncorrelated, the dependence problem that arises when considering a spatial estimator for the variogram or the covariogram is overcome by the use of this spectral estimator. However, Whittle estimation has some problems for stochastic processes in dimension greater or equal to two. In this case (under increasing domain asymptotics), the parametric estimator has a bias of order $N^{-1 / d}$, where $N$ denotes the number of data and $d$ is the dimension of the space. Therefore, using Whittle estimation in the spatial statistical context would not give good results. Some authors have proposed different modifications of Whittle's method, considering adapted versions of the periodogram (Guyon, 1982; Dahlhaus and Künsch, 1987; Robinson and Vidal Sanz, 2006). Our approach does not try to modify the periodogram, but the target function against which it is compared.

We adapt Whittle estimation method in order to get consistent parametric estimators and also in order to mitigate the aliasing problem that occurs when working with discrete-sampled continuous processes in the spectrum. We compare the results of our estimator with the other spectral estimators and also with other methods, more frequently used in the spatial statistical literature, such as WLS, ML and REML methods.

This paper is organized as follows. In section 2, we provide some basics on spectral methods and we also introduce spatial and spectral estimation methods for the covariogram and the spectral density, respectively. The new estimator based on the FFT of biased covariances is introduced in section 3. Section 
4 is devoted to a simulation study where we compare the performance of our method with other spectral and spatial estimators, both for Gaussian and nonGaussian distributed data. In section 5, we include an application to real data, comparing the performance of our estimator, in terms of prediction errors, with other classical approaches. Some discussion is given in section 6 .

\section{Background}

For simplicity, we will set our context in the bidimensional spatial case. Assume that $\left\{Z(\mathbf{s}): \mathbf{s} \in D \subset \mathbb{R}^{2}\right\}$ ( $D$ with fixed positive volume) is a second-order stationary geostatistical process:

$$
E(Z(\mathbf{s}))=\mu ; \quad \operatorname{Cov}(Z(\mathbf{s}), Z(\mathbf{s}+\mathbf{u}))=C(\mathbf{u}) .
$$

Therefore, the process is also intrinsically stationary with variogram $2 \gamma(\mathbf{h})=$ $2(C(\mathbf{0})-C(\mathbf{h}))$ (see e.g. Cressie, 1993). Assuming that $C \in L^{1}\left(\mathbb{R}^{2}\right)$, a continuous covariogram admits a spectral representation:

$$
C(\mathbf{u})=\int_{\mathbb{R}^{2}} e^{i \mathbf{u}^{T} \boldsymbol{\lambda}} f(\boldsymbol{\lambda}) d \boldsymbol{\lambda}
$$

where

$$
f(\boldsymbol{\lambda})=\frac{1}{(2 \pi)^{2}} \int_{\mathbb{R}^{2}} e^{-i \mathbf{u}^{T} \boldsymbol{\lambda}} C(\mathbf{u}) d \mathbf{u}
$$

is a positive function, called the spectral density (being the reciprocal also true; see e.g. Yaglom, 1987).

We will restrict ourselves to the case where the process observations are collected at locations $\mathbf{s}_{\mathbf{j}}$ on a regular grid:

$$
\mathcal{D}=\left\{0, \ldots, \Delta_{1}\left(n_{1}-1\right)\right\} \times\left\{0, \ldots, \Delta_{2}\left(n_{2}-1\right)\right\}
$$

denoting $N=n_{1} n_{2}$ and $\mathbf{s}_{\mathbf{j}}=\left(s_{j_{1}}, s_{j_{2}}\right)^{T}$ with $s_{j_{l}}=\Delta_{l} j_{l} ; \quad j_{l}=0, \ldots, n_{l}-$ $1, \quad l=1,2$. This sampling scheme $\mathcal{D}$ allows us to consider both continuous and discrete spatial processes. In the discrete case (e.g. in time series), $\Delta_{1}$ and $\Delta_{2}$ are set to 1 , and the natural asymptotic framework is an increasing domain design. When dealing with continuous spatial processes, as long as the sample size grows, it is often assumed that the spacing between observations 
tends to zero. This mixture between increasing domain and infilling, called shrinking asymptotics (e.g. Fuentes, 2002), is more natural for studying the asymptotic behaviour of a continuous spatial process. The spectrum of a discrete process can be defined just on the frequency set $\Pi^{2}=[-\pi, \pi] \times[-\pi, \pi]$ instead of considering the whole space $\mathbb{R}^{2}$. However, when working with continuous processes in practice, the spectrum can be recovered only on the set $\Pi_{\Delta}^{2}=\left[-\pi / \Delta_{1}, \pi / \Delta_{1}\right] \times\left[-\pi / \Delta_{2}, \pi / \Delta_{2}\right]$, which is known as the aliasing phenomena. For a certain frequency $\boldsymbol{\lambda}$, the aliased version of the spectral density can be defined as:

$$
f_{\Delta}(\boldsymbol{\lambda})=\sum_{m_{1}=-\infty}^{\infty} \sum_{m_{2}=-\infty}^{\infty} f\left(\lambda_{1}+\frac{2 \pi}{\Delta_{1}} m_{1}, \lambda_{2}+\frac{2 \pi}{\Delta_{2}} m_{2}\right) .
$$

It is important to note that, in the case of discrete processes, as in the time series context, the traditional definition of spectral density corresponds with (3) instead of (2).

In the modelling of the dependence structure of a spatial process, usually a parametric family of covariograms or variograms is chosen. One of most well-known covariogram families is the Matern class (see e.g. Stein, 1999, pp. $48-52)$ :

$$
C_{\theta}(\mathbf{u})=\frac{\sigma^{2}}{2^{\nu-1} \Gamma(\nu)}\left(\frac{\|\mathbf{u}\|}{a}\right)^{\nu} \mathcal{K}_{\nu}\left(\frac{\|\mathbf{u}\|}{a}\right),
$$

where $\mathcal{K}$ is the modified Bessel function of the second type, $\sigma^{2}$ is the variance of the process (sill), $a$ is a scale parameter (proportional to the autocorrelation range) and $\nu$ is a smoothness parameter (related to the differentiability of the process) which determines the shape of the covariogram at small lags. It is important to point out that, under infilling asymptotics, the behaviour of the covariogram near the origin plays a crucial role in spatial prediction (Stein, 1988). The covariogram model (4) corresponds with the following spectral density:

$$
f_{\theta}(\boldsymbol{\lambda})=\frac{\sigma^{2} \Gamma(\nu+1)}{\Gamma(\nu) \pi a^{2 \nu}}\left(\frac{1}{a^{2}}+\|\boldsymbol{\lambda}\|^{2}\right)^{-\nu-1} .
$$

Note that the smoothness parameter $\nu$ controls the rate of decay of the spectral density at high frequencies. Note also that representation (4) is valid for continuous covariograms. In geostatistics, covariogram models that allow a lack of 
continuity at the origin (presence of nugget effect) are traditionally considered in practice assuming that:

$$
C_{\theta}(\mathbf{u})=c_{0} \mathcal{I}_{\{0\}}(\mathbf{u})+C_{\theta}^{0}(\mathbf{u}),
$$

where $c_{0}$ is the nugget effect, $\mathcal{I}_{\{0\}}(\cdot)$ is the indicator function at the origin and $C_{\theta}^{0}(\cdot)$ is the continuous covariogram model. In this case, the variance of the process is given by $\left(c_{0}+\sigma^{2}\right)$ and $\sigma^{2}$ is known as the partial sill. This formulation is not that usual for discrete processes and traditional spectral estimation methods do not consider this aditional variability. In this case, the spectral density for covariance model (6) is given by:

$$
f_{\theta}(\boldsymbol{\lambda})=c_{0}+f_{\theta}^{0}(\boldsymbol{\lambda})
$$

In the next section we briefly revise the classical estimation methods, both from the spatial and the spectral domains. For more details, see Cressie (1993) for spatial estimation and Fuentes (2002) for spectral methods in spatial continuous processes.

\subsection{Estimation in the spatial domain}

One of the most widely used methods for dependence modeling in geostatistics consists in adjusting a parametric variogram model $\gamma_{\theta}(\cdot)$ to a set of pilot estimators $\left\{\hat{\gamma}\left(\mathbf{u}_{i}\right), i=1, \ldots, K\right\}$, using a Weighted Least Squares criterion (WLS):

$$
\hat{\theta}=\arg \min _{\theta} \sum_{i=1}^{K} \omega_{i}\left(\gamma_{\theta}\left(\mathbf{u}_{i}\right)-\hat{\gamma}\left(\mathbf{u}_{i}\right)\right)^{2} .
$$

As a pilot estimator, it is usual to consider the classical variogram estimator:

$$
\hat{\gamma}(\mathbf{u})=\frac{1}{2|N(\mathbf{u})|} \sum_{N(\mathbf{u})}\left(Z\left(\mathbf{s}_{\mathbf{i}}\right)-Z\left(\mathbf{s}_{\mathbf{j}}\right)\right)^{2},
$$

where $N(\mathbf{u})=\left\{(\mathbf{i}, \mathbf{j}): \mathbf{s}_{\mathbf{i}}-\mathbf{s}_{\mathbf{j}}=\mathbf{u}\right\}$ and $\|N(\mathbf{u})\|$ denote the number of contributing pairs at lag $\mathbf{u}$. Following the advice of Journel and Huijbregts (1978, p.74), the fit of a valid model is usually done up to half the maximum possible lag and considering only pilot estimations with at least 30 contributing pairs (i.e. $\|N(\mathbf{u})\| \geq 30)$. The weights $\omega_{i}$ are usually chosen following the idea proposed by Cressie (1985), i.e., $\omega_{i}=\left\|N\left(\mathbf{u}_{i}\right)\right\| / \gamma_{\theta}\left(\mathbf{u}_{i}\right)$, and proceeding iteratively. 
This criterion has some interesting properties: the larger $\left\|N\left(\mathbf{u}_{i}\right)\right\|$, the higher weight for $\mathbf{u}_{i}$; besides, the smaller variogram value, the higher weight for the corresponding residual, so a good fit of the variogram near the origin is usually obtained with this procedure. It is important to point out that the use of a nonparametric local estimator might improve significantly the efficiency of the estimates (see e.g. Fernández-Casal et al., 2003).

Least squares methods only take into account the (asymptotic) second-order structure of the pilot estimator, and it does not require any additional distributional assumption about the data. This fact makes WLS more robust than ML methods when there is a misspecification in the model (e.g. Carroll and Ruppert, 1982) and they are suitable (although maybe not optimal) even for trans-normal data (see e.g. Cressie, 1993, p.98). In fact, the asymptotic distribution and efficiency of least squares estimators have been studied by Lahiri et al. (2002), considering a shrinking asymptotic framework, where consistency properties and asymptotic normality under quite general assumptions are also proved.

If we assume that the data distribution is normal: $\mathbf{Z} \sim \mathcal{N}(\mathbf{X} \boldsymbol{\beta}, \Sigma)$, where $\mathbf{Z}$ is the vector of observations, $\mathbf{X}$ is the design matrix, $\boldsymbol{\beta}$ is the unknown trend parameter vector and $\Sigma$ denotes the covariance matrix; and we reparametrize the covariance matrix such that $\Sigma=\sigma^{2} V(\theta)$, the Maximum Likelihood (ML) estimators are given by:

$$
\begin{gathered}
\hat{\boldsymbol{\beta}}=\left(\mathbf{X}^{T} V(\theta)^{-1} \mathbf{X}\right)^{-1} \mathbf{X}^{T} V(\theta)^{-1} \mathbf{Z}, \\
\hat{\sigma}^{2}=\frac{1}{N}(\mathbf{Z}-\mathbf{X} \hat{\boldsymbol{\beta}})^{T} V(\theta)^{-1}(\mathbf{Z}-\mathbf{X} \hat{\boldsymbol{\beta}}), \\
\hat{\theta}=\arg \min _{\theta}\left(\frac{N}{2} \ln (2 \pi)+\frac{N}{2} \ln \left(\hat{\sigma}^{2}\right)+\frac{1}{2} \ln |V(\theta)|+\frac{N}{2}\right),
\end{gathered}
$$

Mardia and Marshall (1984) prove the asymptotic consistency of these estimators and their Gaussian limit.

A modification of the classical ML estimation, in order to avoid the effect of trend parameters on the estimation of dependence parameters, is the Restricted Maximum Likelihood method (REML). The idea is to apply a linear filter to 
the observed data obtaining a new data set with distribution independent of $\boldsymbol{\beta}$. In this case:

$$
\begin{gathered}
\hat{\sigma}^{2}=\frac{1}{M}(\mathbf{Z}-\mathbf{X} \hat{\boldsymbol{\beta}})^{T} V(\theta)^{-1}(\mathbf{Z}-\mathbf{X} \hat{\boldsymbol{\beta}}), \\
\hat{\theta}=\arg \min _{\theta}\left(\frac{M}{2} \ln (2 \pi)+\frac{M}{2} \ln \left(\hat{\sigma}^{2}\right)-\frac{1}{2}\left|\mathbf{X}^{T} \mathbf{X}\right|+\frac{1}{2}\left|\mathbf{X}^{T} V(\theta)^{-1} \mathbf{X}\right|+\frac{1}{2} \ln |V(\theta)|+\frac{M}{2}\right),
\end{gathered}
$$

where $M=N-p-1$ and $p+1$ is the dimension of $\beta$ ( $p=0$ for stationary processes). Estimations obtained by REML are expected to be more efficient when data distribution is close to normality, the parametric model for the covariance is well specified and the number of data is big enough. However, it is still unclear whether REML outperforms other simpler methods such as WLS.

\subsection{Estimation in the spectral domain}

The periodogram of a continuous spatial process (see Fuentes, 2002) is given by:

$$
I\left(\boldsymbol{\lambda}_{\mathbf{k}}\right)=\frac{\Delta_{1} \Delta_{2}}{(2 \pi)^{2} N}\left|\sum_{\mathbf{s}_{\mathbf{j}} \in \mathcal{D}} Z\left(\mathbf{s}_{\mathbf{j}}\right) e^{-i \boldsymbol{\lambda}_{\mathbf{k}}^{T} \mathbf{s}_{\mathbf{j}}}\right|^{2},
$$

and it is usually computed at the Fourier frequencies $\boldsymbol{\lambda}_{\mathbf{k}}=\left(\lambda_{k_{1}}, \lambda_{k_{2}}\right)$, where:

$$
\lambda_{k_{l}}=\frac{2 \pi k_{l}}{\Delta_{l}\left(2 n_{l}-1\right)} ; \quad k_{l}=0, \pm 1, \ldots, \pm\left(n_{l}-1\right), \quad l=1,2,
$$

in order to avoid leakage problems (and to allow the calculation of the sample covariances inverting the periodogram). From a computational point of view, the advantage of this estimator is that it can be easily obtained using the FFT (by zero padding). In addition, from a statistical point of view, the main advantage is that values at different Fourier frequencies are asymptotically uncorrelated (see Brillinger, 1981, for discrete processes under increasing domain asymptotics and Fuentes, 2002, for continuous processes under shrinking asymptotics). The main drawback of the periodogram is its lack of consistency as an estimator of the spectral density.

This estimator can be also written in terms of the sample covariances:

$$
I\left(\boldsymbol{\lambda}_{\mathbf{k}}\right)=\frac{\Delta_{1} \Delta_{2}}{(2 \pi)^{2}} \sum_{\mathbf{u}_{\mathbf{j}} \in \mathcal{U}} \hat{C}(\mathbf{u}) e^{-i \boldsymbol{\lambda}_{\mathbf{k}}^{T} \mathbf{u}_{\mathbf{j}}}
$$


where:

$$
\mathcal{U}=\left\{\mathbf{u}_{\mathbf{j}}=\left(u_{j_{1}}, u_{j_{2}}\right)^{T} ; u_{j_{l}}=j_{l} \Delta_{l}, \quad j_{l}=0, \pm 1, \ldots, \pm\left(n_{l}-1\right), l=1,2\right\},
$$

and the sample covariances, assuming for simplicity axial symmetry and zero mean, are given by:

$$
\hat{C}\left(\mathbf{u}_{\mathbf{j}}\right)=\frac{1}{N} \sum_{k_{1}=0}^{n_{1}-\left|j_{1}\right|} \sum_{k_{2}=0}^{n_{2}-\left|j_{2}\right|} Z\left(\mathbf{s}_{\mathbf{k}}\right) Z\left(\mathbf{s}_{\mathbf{k}+|\mathbf{j}|}\right) .
$$

Sample covariances given by (9) are not unbiased estimators of the covariance $C$, and this bias will have a non-negligible effect on density estimators based on (8).

Whittle estimation method (introduced in Whittle, 1954, for discrete processes) is based on the approximation to the Gaussian log-likelihood and it uses the periodogram as a pilot estimator of the spectral density. For a parametric spectral density function $f_{\theta} \in \mathcal{F}_{\theta \in \Theta}$, Whittle's estimator is obtained as:

$$
\hat{\theta}=\arg \min _{\theta} L\left(f_{\theta}, I\right),
$$

where $L\left(f_{\theta}, I\right)$ denotes Whittle's log-likelihood function:

$$
L\left(f_{\theta}, I\right)=\int_{\Pi_{\Delta}^{2}}\left(\log f_{\theta}(\lambda)+\frac{I(\lambda)}{f_{\theta}(\lambda)}\right) d \lambda .
$$

This function can be interpreted as the Kullback-Leibler discrepancy between the periodogram $I$ and the spectral density $f_{\theta}$. In practice, a discretized version of this distance is used, replacing the integral by a sum over the Fourier frequencies.

In the case of Gaussian processes, under general conditions, the periodogram can be written as:

$$
I\left(\boldsymbol{\lambda}_{\mathbf{k}}\right)=f_{\Delta}\left(\boldsymbol{\lambda}_{\mathbf{k}}\right) W_{\mathbf{k}}+R_{N}^{\Delta}\left(\boldsymbol{\lambda}_{\mathbf{k}}\right)
$$

with $W_{\mathbf{k}}$ i.i.d. $\operatorname{Exp}(1)$ (standard exponential distribution) and $R_{N}^{\Delta}$ being an asymptotically negligible term. Note that, in case of non-Gaussian processes, this representation holds asymptotically. Whittle estimator is obtained maximizing the likelihood associated with (11), ingnoring the residual part $R_{N}^{\Delta}$. 
Note also that this is equivalent to the maximum likelihood estimator proposed by Fan and Zhang (2004), in the time series context, based on the additive regression model obtained applying logarithms in (11).

As it has been already mentioned, Whittle estimator has a bias of order $N^{-1 / d}$, which is of the same order of the standard deviation in $d=2$ and of a larger order for $d>2$. In order to obtain a consistent estimator, Guyon (1982) replaces the periodogram in (10) by an unbiased version, considering unbiased sample covariances given by:

$$
\tilde{C}\left(\mathbf{u}_{\mathbf{j}}\right)=\sum_{k_{1}=0}^{n_{1}-\left|j_{1}\right|} \sum_{k_{2}=0}^{n_{2}-\left|j_{2}\right|} \frac{Z\left(\mathbf{s}_{\mathbf{k}}\right) Z\left(\mathbf{s}_{\mathbf{k}+\mid \mathbf{j}}\right)}{\left(n_{1}-\left|j_{1}\right|\right)\left(n_{2}-\left|j_{2}\right|\right)} .
$$

Although this approach provides asymptotically consistent estimators, it may present some undesirable features. One of the drawbacks is that $\tilde{C}$ may not be positive definite, which implies that the estimated spectral density may take negative values. Besides, the interpretability of Whittle's estimator as a minimum distance one, is also lost. Dahlhaus and Künsch (1987) prove that the inconsistency problem can be also solved tapering the data before constructing the periodogram. The tapered periodogram is then defined as:

$$
I^{t a p}\left(\boldsymbol{\lambda}_{\mathbf{k}}\right)=\frac{\Delta_{1} \Delta_{2}}{(2 \pi)^{2} H}\left|\sum_{\mathbf{s}_{\mathbf{j}} \in D} h\left(\mathbf{s}_{\mathbf{j}}\right) Z\left(\mathbf{s}_{\mathbf{j}}\right) e^{-i \mathbf{s}_{\mathbf{j}}^{T} \boldsymbol{\lambda}_{\mathbf{k}}}\right|^{2},
$$

where $h$ is the taper function and $H=\sum_{\mathbf{s}_{\mathbf{j}}} h^{2}\left(\mathbf{s}_{\mathbf{j}}\right)$. There are several proposals for tapers (see Priestley, 1981, pp. 561-562) in time series, and for higher dimensions, tapers are usually obtained as tensor products of one-dimensional functions. More recently, Robinson and Vidal Sanz (2006) proved that this edge-effect bias can be also corrected modifying the periodogram by introducing smooth sample covariances:

$$
I^{s m}\left(\boldsymbol{\lambda}_{\mathbf{k}}\right)=\frac{\Delta_{1} \Delta_{2}}{(2 \pi)^{2}} \sum_{\mathbf{u}_{\mathbf{j}} \in \mathcal{U}} K\left(\mathbf{u}_{\mathbf{j}}\right) \hat{C}\left(\mathbf{u}_{\mathbf{j}}\right) e^{-i \mathbf{u}_{\mathbf{j}}^{T} \boldsymbol{\lambda}},
$$

where $K$ is a weight function, usually obtained as tensor product of onedimensional weights. Introducing this weight function, the contributions of the tails in the covariance is reduced. 
Some examples of taper and kernel functions are the multiplicative-cosine taper and the Parzen kernel (see Figures 1 and 2):

- The $m$-cosine multiplicative taper, $h\left(\mathbf{s}_{\mathbf{j}}\right)=h_{n_{1}, m_{1}}\left(j_{1}\right) h_{n_{2}, m_{2}}\left(j_{2}\right)$ where:

$$
h_{n, m}(j)= \begin{cases}\frac{1}{2}\left(1-\cos \left(\frac{\pi(j-1 / 2)}{m}\right)\right), & 1 \leq j \leq m, \\ 1, & m+1 \leq j \leq n-m, \\ h_{n, m}(n-j+1), & n-m+1 \leq j \leq n .\end{cases}
$$

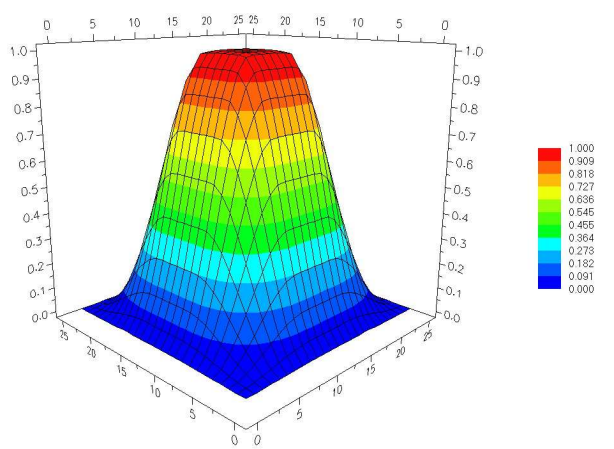

Figure 1: Multiplicative cosine taper with $m_{l}=5, n_{l}=51, l=1,2$.

- The multiplicative Parzen kernel $K\left(\mathbf{u}_{\mathbf{j}}\right)=k_{n_{1}, m_{1}}\left(j_{1}\right) k_{n_{2}, m_{2}}\left(j_{2}\right)$ where:

$$
\begin{gathered}
k_{n, m}(j)=\kappa\left(\frac{j-(n+1) / 2}{(n+1) / 2-m}\right), \\
\kappa(\omega)= \begin{cases}1+6\left(|\omega|^{3}-\omega^{2}\right), & |\omega| \leq 1 / 2, \\
2\left(1-|\omega|^{3}\right), & 1 / 2<|\omega| \leq 1, \\
0, & \text { otherwise. }\end{cases}
\end{gathered}
$$

The asymptotic properties of tapered and smooth estimators in the continuous case (under shrinking asymptotics) can be found in Crujeiras and Fernández-Casal (2008) (see also Robinson, 2007, for discrete multidimensional processes). In practice, these estimators may present some problems. For instance, it is not clear how to chose the suitable tapering or the smoothing functions. In some case, even for large samples, we may find a noticeable bias in the estimation provided by (12) and (13) as it happens for spatial data with a long dependence range. Notice also that, when smoothing the covariances, the result may not be positive definite, providing negative estimations for the spectral density. 


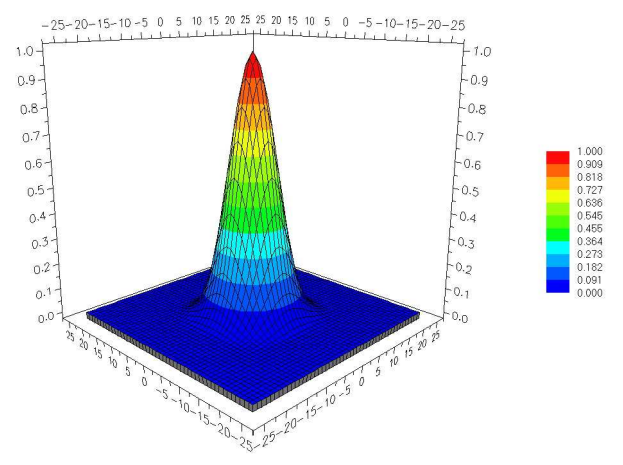

Figure 2: Parzen multiplicative kernel with $m_{l}=5, n_{l}=51, l=1,2$.

\section{$3 \quad$ FFT of biased covariances}

As we have already seen, the periodogram (or any of the modified versions introduced) is not a efficient estimator of the spectral density. This lack of efficiency comes from two main sources: the use of biased covariance estimators and the truncation error in the approximation of the spectral density. Truncation may pose a problem if the autocorrelation range is large compared with the data range, that is, the covariogram takes values different from zero for lags larger than $\Delta_{l}\left(n_{l}-1\right)$. In this case, a non-negligible bias is expected in this type of estimators. In the continuous case, we have the additional problem of aliasing, which gets worse if the spacing between observations is not small enough.

In expression (8) we observe that the periodogram is the Discrete Fourier Transform of the sample covariances (9), which are not unbiased estimators of the covariance function at lags $\mathbf{u}_{\mathbf{j}}$. From this expression, it is easy to see that, the expected value of the periodogram at a certain frequency is given by:

$$
E\left(I\left(\boldsymbol{\lambda}_{\mathbf{k}}\right)\right)=\tilde{f}_{\Delta}\left(\boldsymbol{\lambda}_{\mathbf{k}}\right)=\frac{\Delta_{1} \Delta_{2}}{(2 \pi)^{2}} \sum_{\mathbf{u}_{\mathbf{j}} \in \mathcal{U}} \prod_{l=1}^{2}\left(1-\frac{\left|j_{l}\right|}{n_{l}}\right) C\left(\mathbf{u}_{\mathbf{j}}\right) e^{-i \mathbf{u}_{\mathbf{j}}^{T} \boldsymbol{\lambda}_{\mathbf{k}}},
$$

which may differ notably from the true spectral density $f\left(\boldsymbol{\lambda}_{\mathbf{k}}\right)$. The spectral density and biased covariances FFT surfaces (in logarithmic scale), for a Matern model with parameters $\sigma^{2}=10, a=10$ and $\nu=0.5$ (which corresponds to an exponential covariance model) are shown in Figure 3. We can observe important differences between both surfaces, more remarkable for high frequencies, 
corresponding to covariances at small distances.

$\ln \left(f\left(\boldsymbol{\lambda}_{\mathbf{k}}\right)\right)$

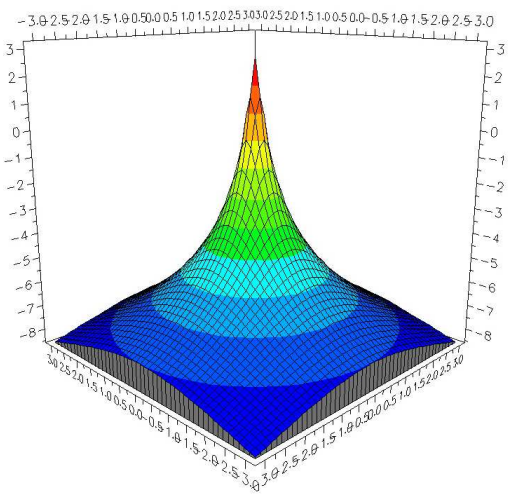

$\ln \left(\tilde{f}_{\Delta}\left(\boldsymbol{\lambda}_{\mathbf{k}}\right)\right)$

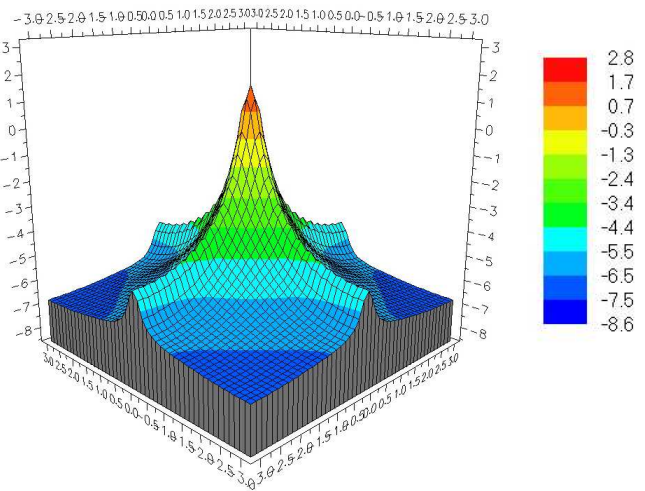

Figure 3: Spectral density and biased covariances FFT (14) surfaces, in logarithmic scale, corresponding to a Matern model (5) with parameters $\sigma^{2}=1.0$, $a=10$ and $\nu=0.5$ and a sample with $N=25 \times 25$ and $\Delta_{1}=\Delta_{2}=1$.

Bearing in mind the previous comments, instead of considering the spectral density as a target for the periodogram, it could be preferable to replace $f_{\theta}$ in (10) by the expected value of the periodogram (14). Proceeding in this way, the new parametric estimator is obtained as:

$$
\tilde{\theta}=\arg \min _{\theta} L\left(\tilde{f}_{\Delta, \theta}, I\right),
$$

where $\tilde{f}_{\Delta, \theta}$ is the expected value of the periodogram in (14) considering a parametric covariance function $C_{\theta}$. If we denote by $f_{\theta_{0}}$ the true spectral density, the new estimator (15) is consistent under some regularity assumptions on the spectral density function and on the design parameters $n_{l}$ and $\Delta_{l}, l=1,2$ (see discussion for further details).

In practice, the estimator (15) is obtained by minimizing a discrete version of $L\left(\tilde{f}_{\theta}, I\right)$. Note that the periodogram can be written as:

$$
I\left(\boldsymbol{\lambda}_{\mathbf{k}}\right)=\tilde{f}_{\Delta}\left(\boldsymbol{\lambda}_{\mathbf{k}}\right) W_{\mathbf{k}},
$$

with $W_{\mathbf{k}}$ i.d. $\operatorname{Exp}(1)$ asymptotically independent. The Whittle-type estimator is also obtained maximizing the corresponding likelihood as:

$$
\tilde{\theta}=\arg \min _{\theta} \sum_{\mathbf{k}}\left(\log \tilde{f}_{\Delta, \theta}\left(\boldsymbol{\lambda}_{\mathbf{k}}\right)+\frac{I\left(\boldsymbol{\lambda}_{\mathbf{k}}\right)}{\tilde{f}_{\Delta, \theta}\left(\boldsymbol{\lambda}_{\mathbf{k}}\right)}\right) .
$$


This estimator will be named FFTW along the paper. All bias sources that appear in the periodogram as an estimator of the spectral density, including the aliasing phenomena for continuous processes, are taking into account in this formulation.

\section{Simulation results}

In the numerical study the proposed estimator is compared with the methods traditionally used in the modeling of spatial dependency (see Section 2). First, spectral estimation methods are considered: Whittle estimation and modified versions by tapering and smoothing covariances methods (and combination of both approaches); secondly, the FFTW estimator is compared with the spatialscale estimators: WLS, ML and REML methods.

\subsection{Comparison with spectral estimation methods}

In this case stationary gaussian spatial processes were simulated on regular grids of sizes $N=8 \times 8$ and $N=15 \times 15$, with unit spacing $\left(\Delta_{1}=\Delta_{2}=1\right)$. The Matern covariance model (4) was used as underlying covariogram with parameters: $c_{0}=0$ (the nugget effect was fixed to cero), $\sigma^{2}=1$, smoothness parameter $\nu=0.5$ and scale parameters $a=3$ and $a=5$ corresponding to the diferent grid sizes in order to make results comparable. For each process, 1000 samples were generated. In each simulation, parameter estimates were obtained using the FFTW, classical Whittle estimation and the modified versions by tapering, smoothing and combinations of both. Multiplicative cosine tapers and Parzen kernels for covariance smoothing were used in the calculation of the modified periodograms (Section 2.2).

The boxplots of the parameter estimates for the case of $N=8 \times 8$, and the true parameter values, are shown in Figure 4; a summary of these values is shown in Table 1 (note that in this case the nugget effect was set to zero). We can see that the results obtained by Whittle estimation and modified versions (even for different bandwidth $m$ parameters) are clearly not satisfactory. Overestimations of the variance and the scale parameter and underestimations of the 
smoothing parameter were obtained with these estimators (reaching the parameter estimation limits, fixed in this case to $1.0 \leq \hat{a} \leq 50.0$ and $0.1 \leq \hat{\nu} \leq 2.0$ ). The estimates obtained with the FFTW estimator proposed in this work are always those nearest the true value.

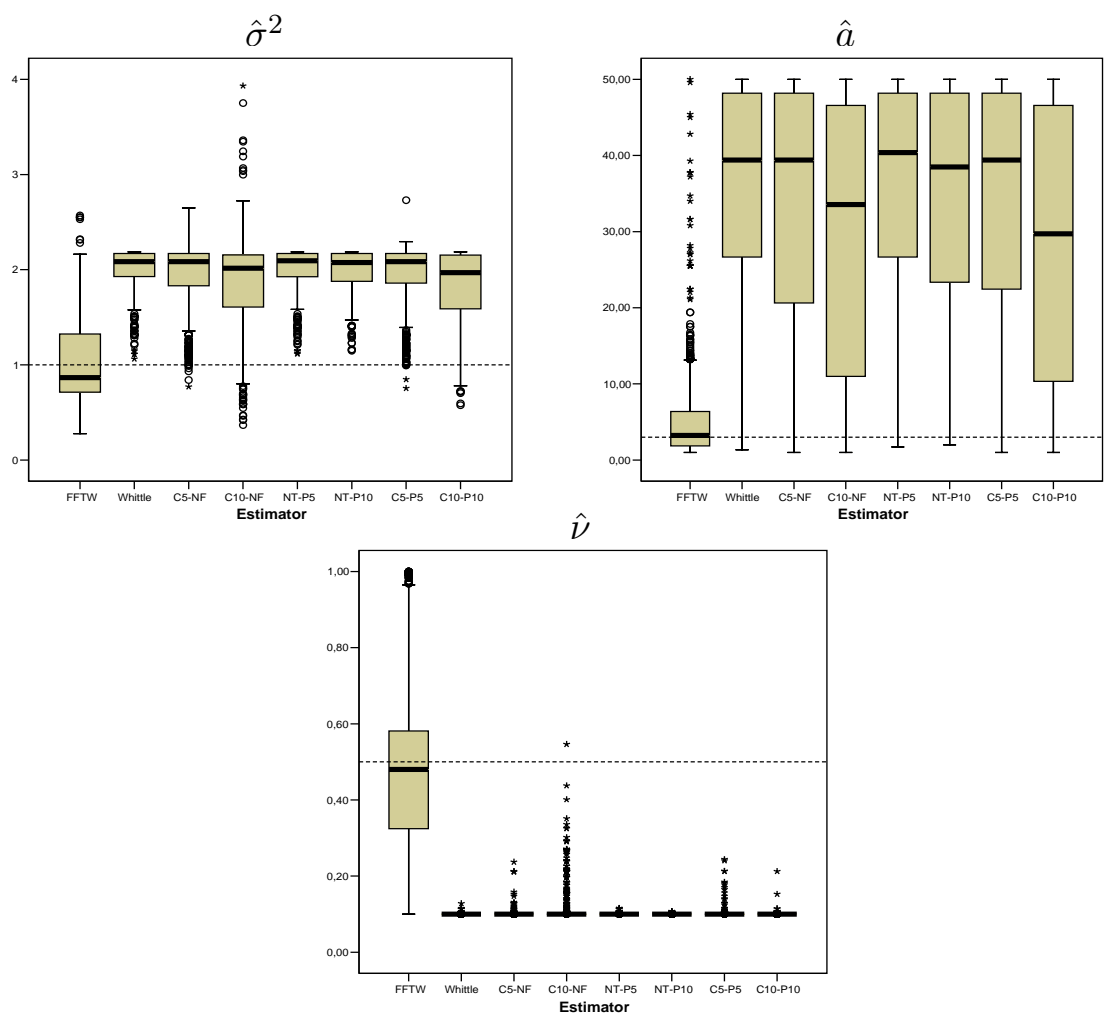

Figure 4: Parameter estimates, for the case of $N=8 \times 8$, obtained with the different methods. FFTW; Whittle estimator; $\mathrm{Cm}$-NF: cosine tapered data and no smoothed covariances; NT-P $m$ : no tapered data and smoothed covariances with Parzen kernel; $\mathrm{Cm}$-Pm: tapered data and smoothed covariances. In all cases, $m=5,10$ is the bandwith parameter. The dashed lines represent the true parameter values.

The results obtained for the case of a $N=15 \times 15$ regular grid are shown in Figure 5 and Table 2. The behaviour of the different estimators is very similar to that for the smaller grid. Apparently, there is a slightly improvement in the estimates obtained with the classical spectral methods when the number of data is larger, but still far from the performance of the FFTW method (this be- 


\begin{tabular}{|c|c|c|c|c|}
\hline Parameter & Estimator & Mean & Median & St.Dev. \\
\hline \multirow{8}{*}{$\sigma^{2}=1$} & FFTW & 0.9742 & 0.8668 & 0.3792 \\
\hline & Whittle & 2.0149 & 2.0850 & 0.2071 \\
\hline & $\mathrm{C} 5-\mathrm{NF}$ & 1.9627 & 2.0846 & 0.2867 \\
\hline & C10-NF & 1.8288 & 2.0151 & 0.4856 \\
\hline & NT-P5 & 2.0141 & 2.0940 & 0.2097 \\
\hline & NT-P10 & 1.9957 & 2.0754 & 0.2097 \\
\hline & C5-P5 & 1.9708 & 2.0850 & 0.2846 \\
\hline & C10-P10 & 1.8015 & 1.9696 & 0.4048 \\
\hline \multirow{8}{*}{$a=3$} & FFTW & 5.1752 & 3.2267 & 6.0553 \\
\hline & Whittle & 36.3931 & 39.4040 & 13.5633 \\
\hline & $\mathrm{C} 5-\mathrm{NF}$ & 34.2702 & 39.4040 & 15.5100 \\
\hline & C10-NF & 29.0414 & 33.5559 & 18.3553 \\
\hline & NT-P5 & 36.4145 & 40.3717 & 13.6404 \\
\hline & NT-P10 & 34.8582 & 38.5027 & 13.8949 \\
\hline & C5-P5 & 34.7961 & 39.4040 & 15.4004 \\
\hline & C10-P10 & 27.2345 & 29.7199 & 18.1122 \\
\hline \multirow{8}{*}{$\nu=0.5$} & FFTW & 0.4952 & 0.4804 & 0.2089 \\
\hline & Whittle & 0.1003 & 0.1000 & 0.0014 \\
\hline & $\mathrm{C} 5-\mathrm{NF}$ & 0.1011 & 0.1000 & 0.0084 \\
\hline & C10-NF & 0.1097 & 0.1000 & 0.0376 \\
\hline & NT-P5 & 0.1003 & 0.1000 & 0.0013 \\
\hline & NT-P10 & 0.1002 & 0.1000 & 0.0006 \\
\hline & C5-P5 & 0.1015 & 0.1000 & 0.0103 \\
\hline & C10-P10 & 0.1003 & 0.1000 & 0.0040 \\
\hline
\end{tabular}

Table 1: Summary statistics of parameter estimates, for the case of $N=8 \times$ 8, obtained with the different methods. FFTW; Whittle estimator; $\mathrm{Cm}$-NF: cosine tapered data and no smoothed covariances; NT-P $m$ : no tapered data and smoothed covariances with Parzen kernel; $\mathrm{Cm}$-Pm: tapered data and smoothed covariances. In all cases, $m=5,10$ is the bandwith parameter. 
haviour continues when the number of data increases, but even for $N=50 \times 50$ the estimates obtained with the classical spectral methods are quite unsatisfactory). Therefore, though the sample size being large, the classical spectral estimators may be unsuitable for the estimation of the covariogram parameters in a multidimensional case.

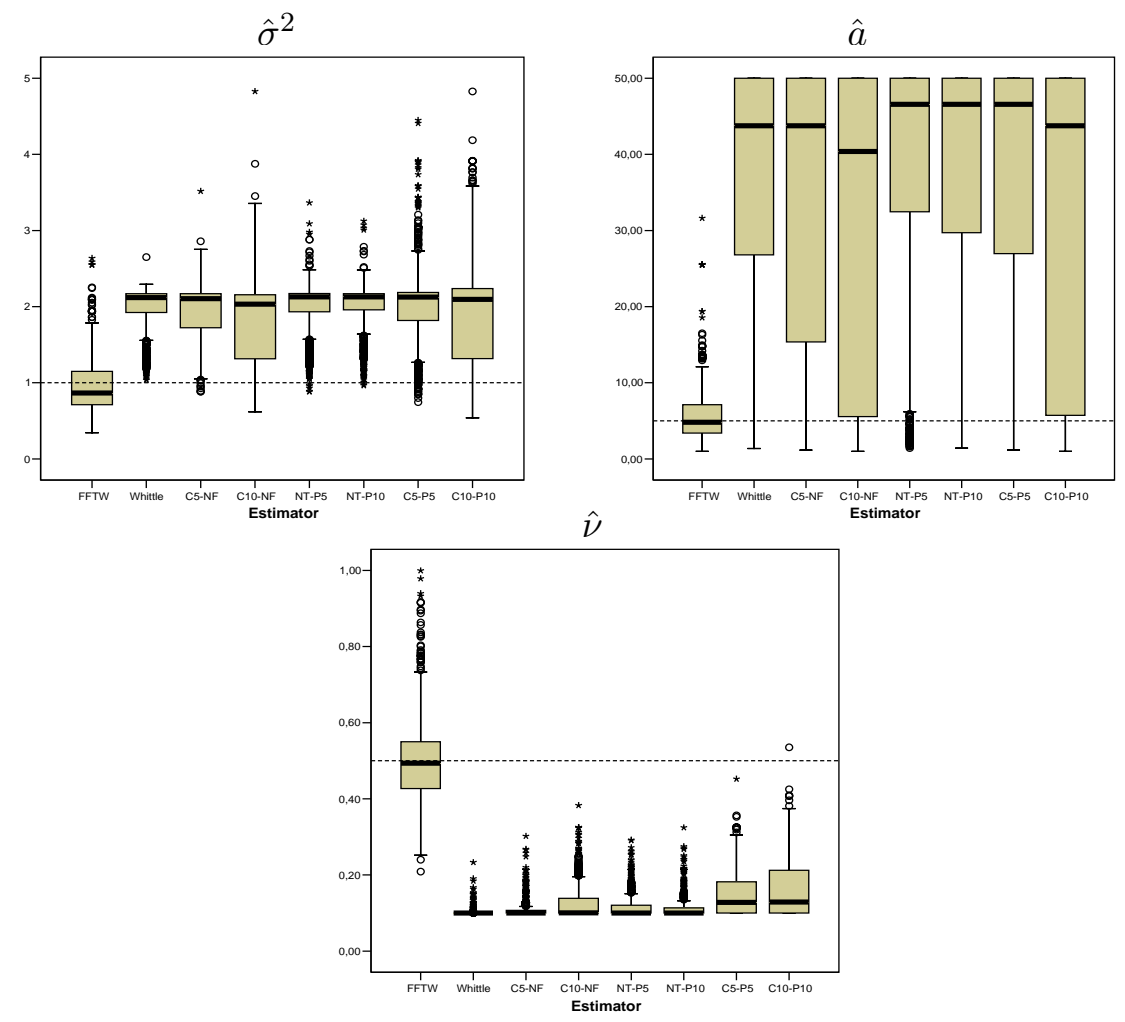

Figure 5: Parameter estimates, for the case of $N=15 \times 15$, obtained with the different methods. FFTW; Whittle estimator; $\mathrm{Cm}$-NF: cosine tapered data and no smoothed covariances; NT-Pm: no tapered data and smoothed covariances with Parzen kernel; $\mathrm{Cm}$-Pm: tapered data and smoothed covariances. In all cases, $m=5,10$ is the bandwith parameter. The dashed lines represent the true parameter values.

\subsection{Comparison with spatial estimation methods}

In order to compare the performance of FFTW with the traditional estimation methods in the spatial scale (WLS, ML and REML), stationary Gaussian spa- 


\begin{tabular}{|c|c|c|c|c|}
\hline Parameter & Estimator & Mean & Median & St.dev. \\
\hline \multirow{8}{*}{$\sigma^{2}=1$} & FFTW & .9441 & .8666 & .34930 \\
\hline & Whittle & 1.9753 & 2.1201 & .29352 \\
\hline & $\mathrm{C} 5-\mathrm{NF}$ & 1.9155 & 2.1048 & .36858 \\
\hline & C10-NF & 1.8176 & 2.0332 & .50417 \\
\hline & NT-P5 & 1.9979 & 2.1268 & .32480 \\
\hline & NT-P10 & 2.0007 & 2.1274 & .30671 \\
\hline & C5-P5 & 2.0323 & 2.1260 & .56251 \\
\hline & C10-P10 & 1.9681 & 2.0952 & .77743 \\
\hline \multirow{8}{*}{$a=5$} & FFTW & 5.6234 & 4.8268 & 3.36877 \\
\hline & Whittle & 35.9510 & 43.7678 & 15.99615 \\
\hline & C5-NF & 34.4412 & 43.7678 & 18.14121 \\
\hline & C10-NF & 30.5469 & 40.3717 & 20.03465 \\
\hline & NT-P5 & 38.0664 & 46.5740 & 15.86353 \\
\hline & NT-P10 & 37.7292 & 46.5740 & 15.58929 \\
\hline & C5-P5 & 36.9647 & 46.5740 & 17.78216 \\
\hline & C10-P10 & 32.1786 & 43.7678 & 19.97101 \\
\hline \multirow{8}{*}{$\nu=0.5$} & FFTW & .4985 & .4937 & .11543 \\
\hline & Whittle & .1019 & .1001 & .00851 \\
\hline & $\mathrm{C} 5-\mathrm{NF}$ & .1117 & .1001 & .02549 \\
\hline & C10-NF & .1252 & .1007 & .04237 \\
\hline & NT-P5 & .1169 & .1003 & .03215 \\
\hline & NT-P10 & .1135 & .1002 & .02884 \\
\hline & $\mathrm{C} 5-\mathrm{P} 5$ & .1486 & .1278 & .05777 \\
\hline & C10-P10 & .1589 & .1287 & .06931 \\
\hline
\end{tabular}

Table 2: Summary statistics of parameter estimates, for the case of $N=15 \times$ 15, obtained with the different methods. FFTW; Whittle estimator; $\mathrm{Cm}$-NF: cosine tapered data and no smoothed covariances; NT-P $m$ : no tapered data and smoothed covariances with Parzen kernel; $\mathrm{Cm}$-Pm: tapered data and smoothed covariances. In all cases, $m=5,10$ is the bandwidth parameter. 
tial processes were simulated on a regular grid of size $N=15 \times 15$ with unit spacing. Simulations were obtained considering a Matern covariance model with unit variance, nugget effect $c_{0}=0$ and $c_{0}=0.25$ (with partial sill $\sigma^{2}=1$ and $\sigma^{2}=0.75$, respectively), scale parameter $a=5$ and smoothness parameter $\nu=0.5$. In each of the 1000 samples generated for each process, parameter estimates were obtained using the diferent methods. For the WLS fits, the procedure described in Section 2.1 was followed, using the classical variogram estimator to obtain pilot estimates at lags $u=1, \ldots, 10$ and fitting the corresponding variogram model iteratively (combining weighted least squares linear regression with a modified Levenberg-Marquardt algorithm). In the computation of the ML and REML estimates, to avoid the problem of possible multimodality of the likelihood surface, a micro-genetic algorithm was used to minimize the negative log-likelihood (see e.g. Goldberg, 1989). This optimization method was also used in the FFTW estimation.

Boxplots of the parameter estimates are shown in Figure 6, for the case of $c_{0}=0$, and Figure 7 , for the case of $c_{0}=0.25$. Summaries of these values are shown in Tables 3 and 4, respectively. It is quite remarkable the great variability of the estimates obtained with the WLS criterion (though it should be expected a significant improvement if a nonparametric pilot estimator were used). Besides, the partial sill is underestimated with ML and REML when $c_{0}=0$, improving considerably with a $25 \%$ of nugget effect (it seems that an underestimation of the variance could be expected with these methods when the spatial dependence is strong; see also Zimmerman and Zimmerman, 1991, p.87). In general, we could say that the FFTW method is the one that provide the best results in all cases.

\subsection{Non-Gaussian data}

In order to study the robustness of the proposed estimation method with respect to the deviations from normality, some simulations considering stationary spatial process with $\chi^{2}$ and $\log \mathcal{N}$ (log-normal) distributions were also generated. 

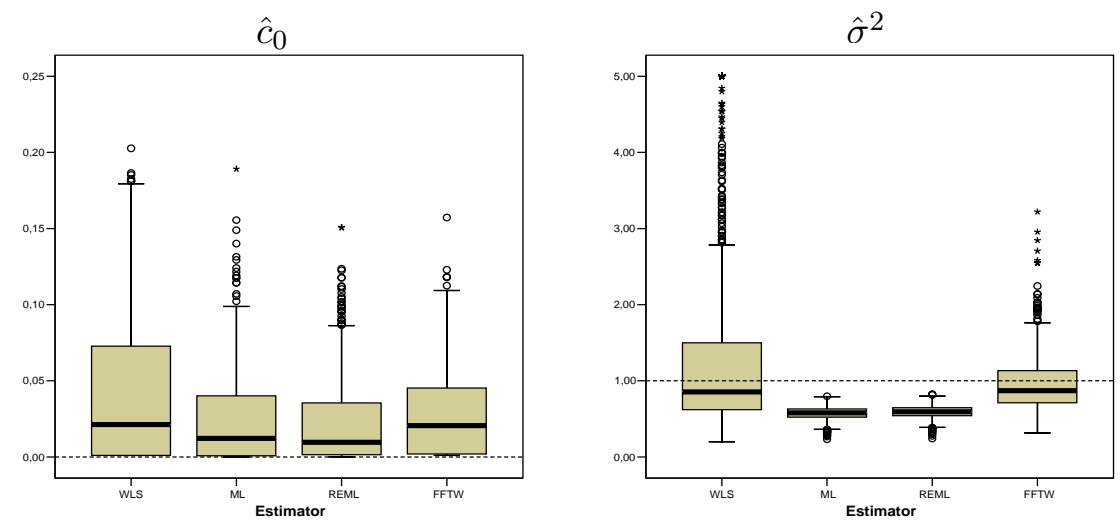

$\hat{a}$
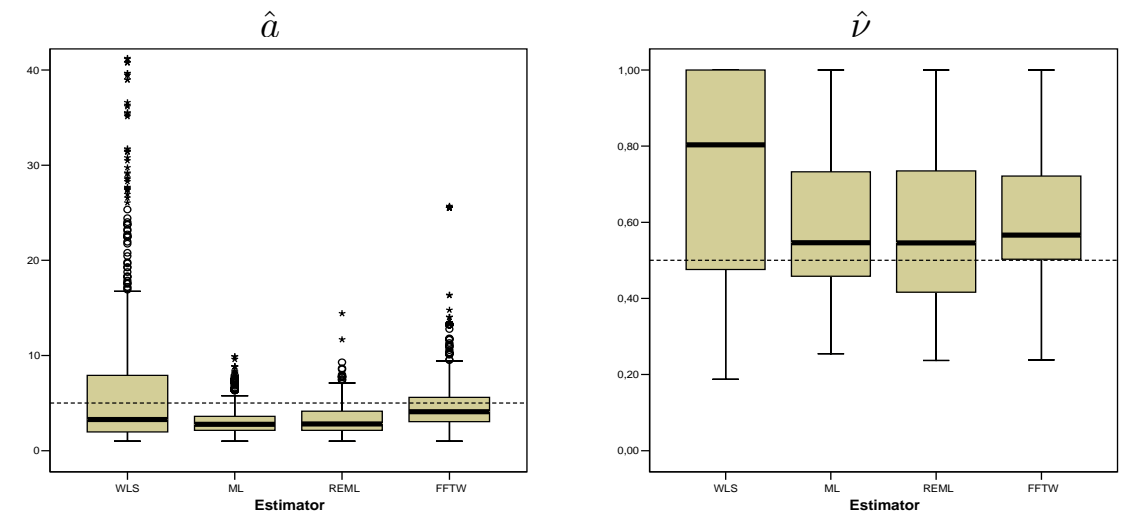

Figure 6: Parameter estimates, for the case of $c_{0}=0$ and $N=15 \times 15$, obtained with the different methods: FFTW, WLS (Weighted Least Squares), ML (Maximum Likelihood) and REML (Restricted Maximum Likelihood). The dashed lines represent the true parameter values.

We can easily simulate $\chi_{1}^{2}$-distributed data with a given covariogram model from a Gaussian process. Given that if $Z(\mathbf{s})=X^{2}(\mathbf{s})$ is a stationary spatial process, where $X(\mathbf{s})$ is Gaussian with covariogram $C_{X}(\mathbf{u})$, then, the covariogram of $Z$ can be obtained as:

$$
C_{Z}(\mathbf{u})=2 C_{X}^{2}(\mathbf{u})
$$

Following the previous observation, 1000 replicates of a $\chi_{1}^{2}$ spatial process were generated on a $10 \times 10$ regular grid with unit spacing, considering a Matern covariogram model with parameters $c_{0}=0.25, \sigma^{2}=0.75, a=4$ and $\nu=0.5$. In each simulation, parameter estimates were obtained in an analogous way to that of the previous section. Results are shown in Figure 8 and some summary statistics are given in Table 5. We can see that, compared with the Gaussian 

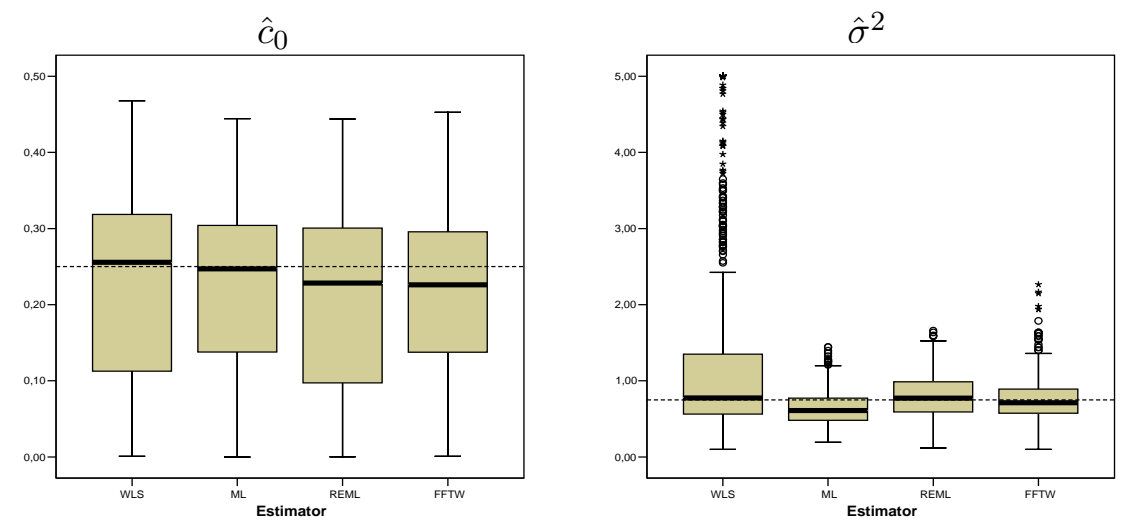

$\hat{a}$
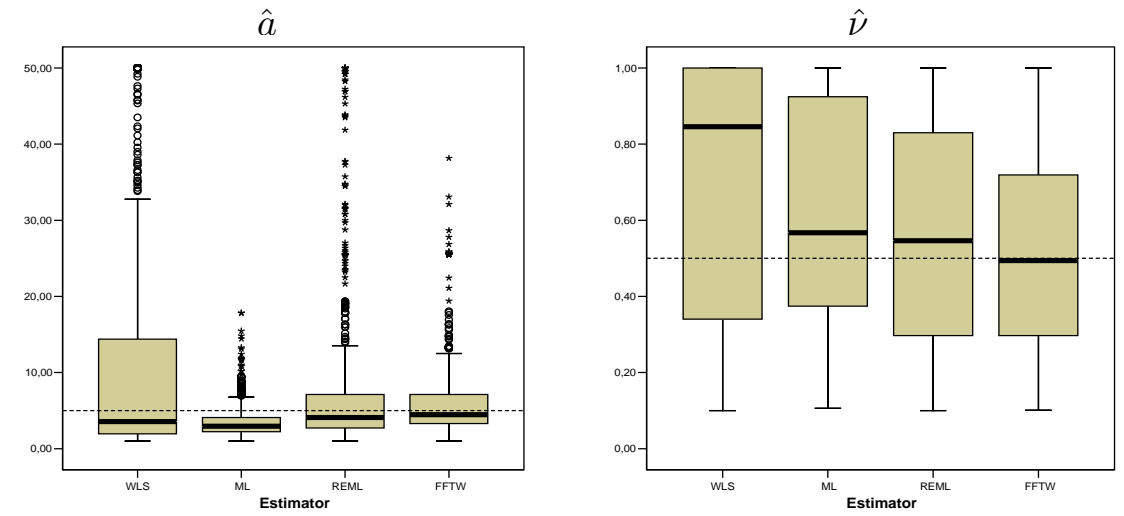

Figure 7: Parameter estimates, for the case of $c_{0}=0.25$ and $N=15 \times 15$, obtained with the different methods: FFTW, WLS (Weighted Least Squares), ML (Maximum Likelihood) and REML (Restricted Maximum Likelihood). The dashed lines represent the true parameter values.

case, the distribution of the estimates is more skewed to the right, with some exceptionally large values especially in the nugget and partial sill estimations. Furthermore, the smoothness parameter is overestimated by all methods. In general, we can say that the REML and FFTW estimators perform better than the others.

It is easy to see that if $Z(\mathbf{s})=\exp (X(\mathbf{s}))$, where $X(\mathbf{s})$ is a stationary Gaussian process with covariogram $C_{X}(\mathbf{u})$, then the corresponding covariogram of $Z$ is given by:

$$
C_{Z}(\mathbf{u})=\mu_{Z}^{2}\left(e^{C_{X}(\mathbf{u})}-1\right)
$$

where $\mu_{Z}=\exp \left(\mu_{X}+C_{X}(\mathbf{0}) / 2\right)$. From this result, 1000 replicates of a lognormal $(\log \mathcal{N})$ process $Z$ were generated on a regular grid of size $N=10 \times 10$, 


\begin{tabular}{|c|cccc|}
\hline Parameter & Estimator & Mean & Median & St.dev. \\
\hline \hline \multirow{3}{*}{$c_{0}=0.0$} & WLS & 0.0399 & 0.0213 & 0.0457 \\
\cline { 2 - 5 } & ML & 0.0239 & 0.0122 & 0.0286 \\
\cline { 2 - 5 } & REML & 0.0219 & 0.0096 & 0.0270 \\
\cline { 2 - 5 }$\sigma^{2}=1.0$ & FFTW & 0.0286 & 0.0205 & 0.0291 \\
\hline \hline \multirow{5}{*}{$a=5$} & WLS & 1.3941 & 0.8554 & 1.2969 \\
\cline { 2 - 5 } & ML & 0.5731 & 0.5795 & 0.0876 \\
\cline { 2 - 5 } & REML & 0.5920 & 0.5941 & 0.0844 \\
\cline { 2 - 5 } & FFTW & 0.9509 & 0.8698 & 0.3751 \\
\hline \hline \multirow{5}{*}{$y=0.5$} & WLS & 9.2473 & 3.2810 & 13.8221 \\
\cline { 2 - 5 } & ML & 3.0708 & 2.7706 & 1.4631 \\
\cline { 2 - 5 } & REML & 3.1799 & 2.8169 & 1.4201 \\
\cline { 2 - 5 } & FFTW & 4.8945 & 4.0865 & 3.1819 \\
\hline \hline \multirow{5}{*}{} & WLS & 0.7365 & 0.8033 & 0.2699 \\
\cline { 2 - 5 } & ML & 0.5991 & 0.5463 & 0.1988 \\
\cline { 2 - 5 } & REML & 0.5873 & 0.5458 & 0.1942 \\
\cline { 2 - 5 } & FFTW & 0.6175 & 0.5662 & 0.1679 \\
\hline
\end{tabular}

Table 3: Summary statistics of parameter estimates, for the case of $c_{0}=0$ and $N=15 \times 15$, obtained with the different estimation methods.

with the same Matern covariogram $\left(c_{0}=0.25, \sigma^{2}=0.75, a=4\right.$ and $\left.\nu=0.5\right)$. Results of the estimations with the different methods are shown in Figure 9 and some summary statistics are given in Table 6. Although the data distribution is less skewed than in the $\chi_{1}^{2}$ case, the estimates of the dependence parameters are very similar. However, in this case, the REML estimates of the partial sill and the scale parameters are much high variable than the obtained with the FFTW estimator. 


\begin{tabular}{|c|cccc|}
\hline Parameter & Estimator & Mean & Median & St.dev. \\
\hline \hline \multirow{3}{*}{$c_{0}=0.25$} & WLS & 0.2196 & 0.2557 & 0.1319 \\
\cline { 2 - 5 } & ML & 0.2193 & 0.2471 & 0.1112 \\
\cline { 2 - 5 } & REML & 0.2017 & 0.2285 & 0.1214 \\
\cline { 2 - 5 }$\sigma^{2}=0.75$ & FFTW & 0.2068 & 0.2261 & 0.1047 \\
\hline \hline \multirow{5}{*}{$a=5$} & WLS & 1.2890 & 0.7761 & 1.2755 \\
\cline { 2 - 5 } & ML & 0.6406 & 0.6089 & 0.2184 \\
\cline { 2 - 5 } & REML & 0.7977 & 0.7737 & 0.2736 \\
\cline { 2 - 5 } & FFTW & 0.7622 & 0.7125 & 0.2780 \\
\hline \hline \multirow{5}{*}{} & WLS & 13.1458 & 3.5664 & 17.8940 \\
\cline { 2 - 5 } & ML & 3.5872 & 2.9485 & 2.1492 \\
\cline { 2 - 5 } & REML & 7.3323 & 4.0925 & 9.1880 \\
\cline { 2 - 5 } & FFTW & 6.0913 & 4.4693 & 4.9116 \\
\hline \hline \multirow{5}{*}{$\nu=0.5$} & WLS & 0.6940 & 0.8459 & 0.3335 \\
\cline { 2 - 5 } & ML & 0.6263 & 0.5673 & 0.2753 \\
\cline { 2 - 5 } & REML & 0.5585 & 0.5463 & 0.2862 \\
\cline { 2 - 5 } & FFTW & 0.5187 & 0.4942 & 0.2511 \\
\hline
\end{tabular}

Table 4: Summary statistics of parameter estimates, for the case of $c_{0}=0.25$ and $N=15 \times 15$, obtained with the different estimation methods.

\section{Application to real data.}

In this section, an example of the application of the proposed method to a set of real data is given. The observations are measurements of the east-west component of wind speed on a grid of $17 \times 17$ spatial positions (regularly spaced, approx. $210 \mathrm{~km}$ ) located in the tropical western Pacific Ocean (the same data set as that analyzed by Cressie and Huang, 1999). Although the whole data set consists of spatio-temporal measuraments, in this case only the observations corresponding to the first time moment were considered. Following an exploratory analysis of the data, it could be seen (e.g. Cressie and Huang, 1999, p.1336) that the stationarity assumption is reasonable. 

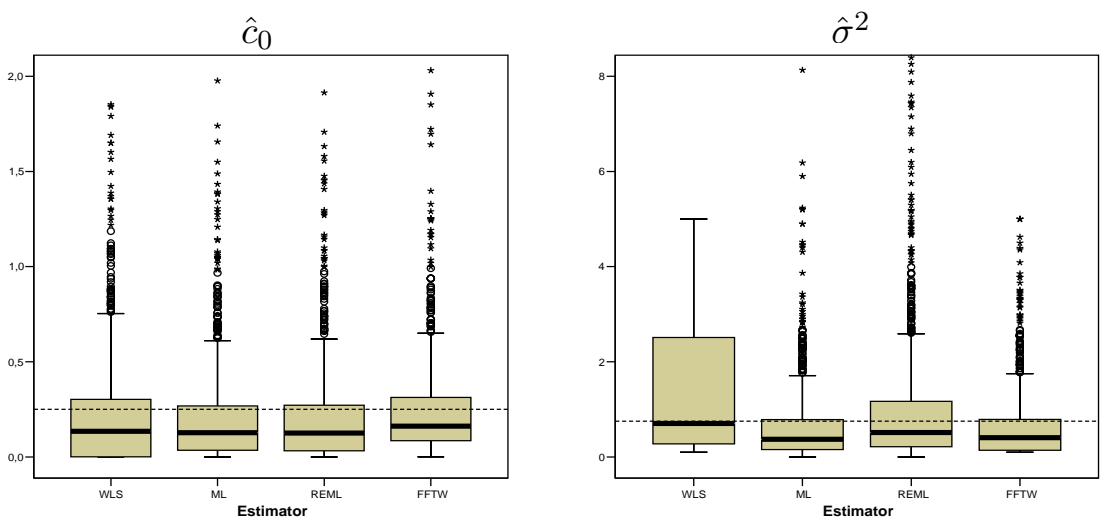

$\hat{a}$
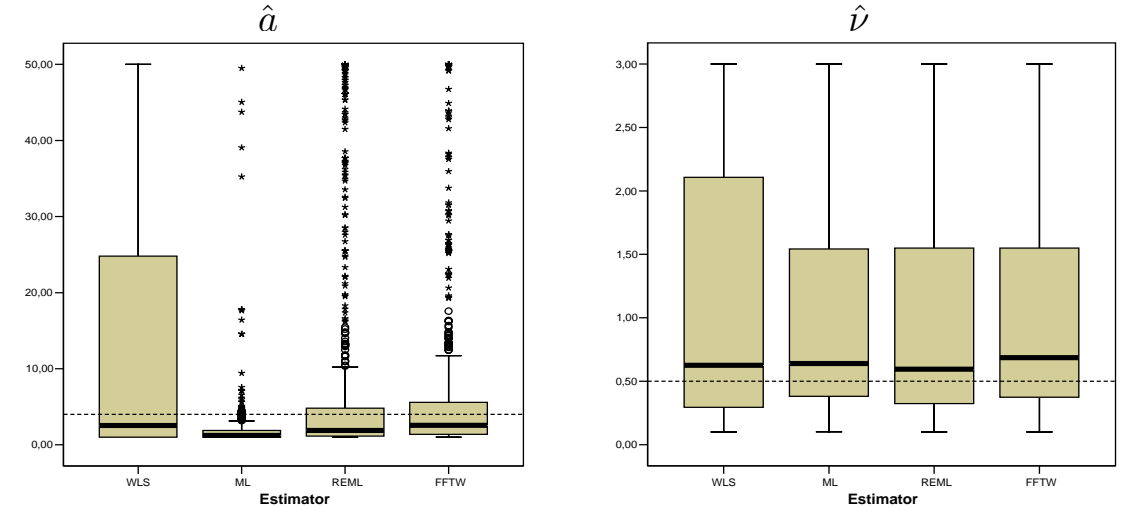

Figure 8: Parameter estimates, for a $\chi_{1}^{2}$-distributed process $(N=10 \times 10)$, with the different estimation methods. The dashed lines represent the true parameter values.

Considering the Matern covariance model given in (5), parameter estimates were obtained by different methods: WLS, ML, REML and FFTW. Parameter estimates are given in Table 5 and plots corresponding to classical variogram estimator and fitted models are shown in Figure 5. We can observe that WLS and FFTW provide quite similar fits, specially at small lags. REML and ML results were also almost identical, with much smaller variance estimates compared with the other methods.

In order to assess if the fitted variogram models adequately described the dependence of the data, the cross-validation technique was used (see, for example, Cressie, 1993, pp. 101-104). For each location $\mathbf{s}_{j}$, using the ordinary kriging method, a predictor $\hat{Z}_{-j}\left(\mathbf{s}_{j}\right)$ and the corresponding mean-squared prediction error $\sigma_{-j}\left(\mathbf{s}_{j}\right)$ were calculated from $\left.\left\{Z\left(\mathbf{s}_{1}\right), \ldots, Z\left(\mathbf{s}_{j-1}\right), Z\left(\mathbf{s}_{j+1}\right), \ldots, Z\left(\mathbf{s}_{n}\right)\right)\right\}$. 


\begin{tabular}{|c|c|c|c|c|}
\hline Parameter & Estimator & Mean & Median & St.dev. \\
\hline \multirow{4}{*}{$c_{0}=0.25$} & WLS & 0.2273 & 0.1352 & 0.3196 \\
\hline & $\mathrm{ML}$ & 0.2094 & 0.1276 & 0.2678 \\
\hline & REML & 0.2111 & 0.1253 & 0.2712 \\
\hline & FFTW & 0.2447 & 0.1614 & 0.2668 \\
\hline \multirow{4}{*}{$\sigma^{2}=0.75$} & WLS & 1.6219 & 0.7031 & 1.8130 \\
\hline & ML & 0.6488 & 0.3724 & 0.8800 \\
\hline & REML & 1.0763 & 0.5147 & 1.5912 \\
\hline & FFTW & 0.6711 & 0.4066 & 0.7766 \\
\hline \multirow{4}{*}{$a=4$} & WLS & 14.4407 & 2.5358 & 19.3168 \\
\hline & ML & 2.0014 & 1.2482 & 3.3586 \\
\hline & REML & 7.3645 & 1.9025 & 13.0387 \\
\hline & FFTW & 6.4833 & 2.5545 & 10.2895 \\
\hline \multirow{4}{*}{$\nu=0.5$} & WLS & 1.1671 & 0.6261 & 1.0952 \\
\hline & $\mathrm{ML}$ & 1.0310 & 0.6390 & 0.8825 \\
\hline & REML & 1.0215 & 0.5947 & 0.9133 \\
\hline & FFTW & 1.0347 & 0.6860 & 0.8686 \\
\hline
\end{tabular}

Table 5: Summary statistics of the parameter estimates, for a $\chi_{1}^{2}$-distributed process $(N=10 \times 10)$, obtained with the different estimation methods.

The efficiency of the predictions was measured by the averaged squared prediction error (ASE):

$$
A S E=\frac{1}{n} \sum_{j=1}^{n}\left(Z\left(\mathbf{s}_{j}\right)-\hat{Z}_{-j}\left(\mathbf{s}_{j}\right)\right)^{2},
$$

and the agreement between the kriging and observed variances was measured by the dimensionless averaged squared error (DASE):

$$
D A S E=\sqrt{\frac{1}{n} \sum_{j=1}^{n}\left(\frac{Z\left(\mathbf{s}_{j}\right)-\hat{Z}_{-j}\left(\mathbf{s}_{j}\right)}{\sigma_{-j}\left(\mathbf{s}_{j}\right)}\right)^{2}},
$$

(this should be close to 1 if there is agreement).

In Table 5, values of ASE and DASE for the different variogram estimators are given. We can observe that the lower ASE values are provided by the FFTW and WLS methods (much better than the given by the ML methods). 

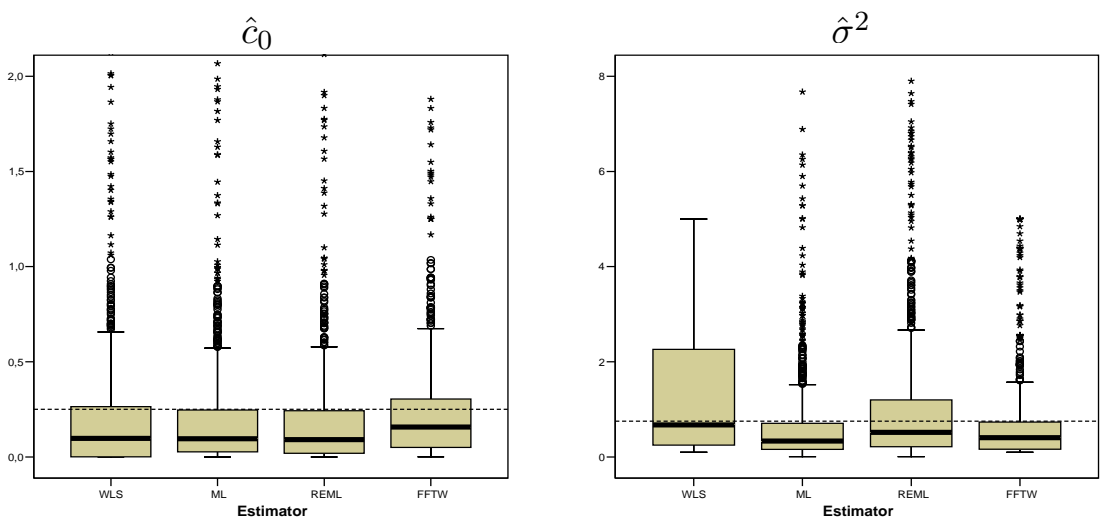

$\hat{a}$
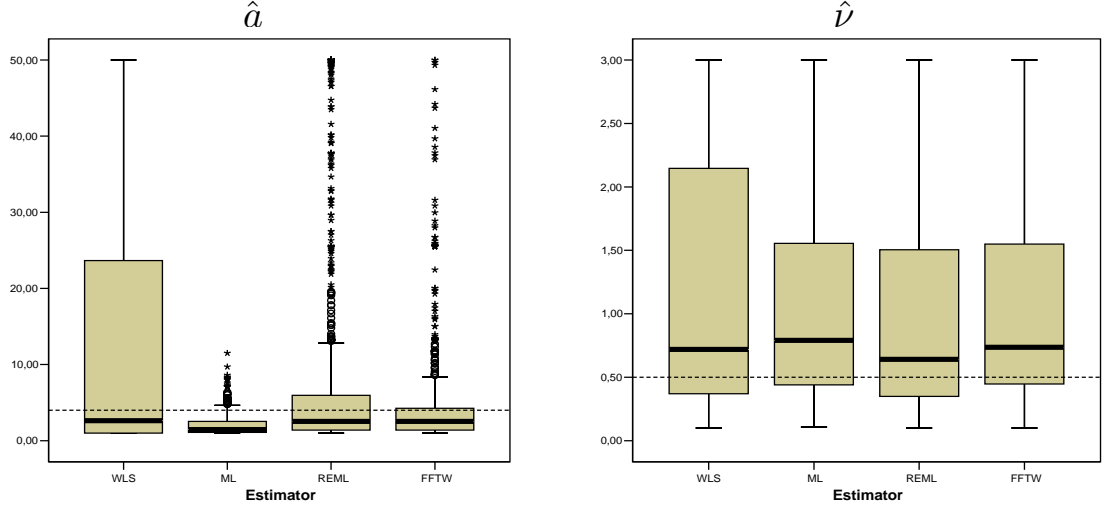

Figure 9: Parameter estimates, for a $\log \mathcal{N}$-distributed process $(N=10 \times 10)$, with the different estimation methods. The dashed lines represent the true parameter values.

Furthermore, the DASE values obtained by all methods are less than 1, reflecting excessively pessimistic estimates of the prediction variances, the FFTW method also got the best results.

\section{Discussion}

In this work, we propose a parametric estimation method for spatial dependence models that can be easily implemented in practice. As the estimation is done in the spectral domain, the inconveniences when applying spatial-domain methods due to the dependence between data are avoided. Our proposal presents a better finite sample behaviour than other spectral approaches, such as the tapered or smoothed periodogram alternatives, since the main bias sources in 


\begin{tabular}{|c|c|c|c|c|}
\hline Parameter & Estimator & Mean & Median & St.dev. \\
\hline \multirow{4}{*}{$c_{0}=0.25$} & WLS & 0.2494 & 0.0976 & 0.5020 \\
\hline & ML & 0.2312 & 0.0958 & 0.4749 \\
\hline & REML & 0.2238 & 0.0908 & 0.4765 \\
\hline & FFTW & 0.2628 & 0.1571 & 0.4762 \\
\hline \multirow{4}{*}{$\sigma^{2}=0.75$} & WLS & 1.5434 & 0.6753 & 1.7494 \\
\hline & ML & 0.6854 & 0.3337 & 1.2130 \\
\hline & REML & 1.1808 & 0.5185 & 2.4349 \\
\hline & FFTW & 0.6759 & 0.4061 & 0.8852 \\
\hline \multirow{4}{*}{$a=4$} & WLS & 14.4472 & 2.6418 & 19.3626 \\
\hline & ML & 1.9663 & 1.4696 & 1.3175 \\
\hline & REML & 8.0209 & 2.5328 & 13.1271 \\
\hline & FFTW & 4.5610 & 2.5373 & 6.4999 \\
\hline \multirow{4}{*}{$\nu=0.5$} & WLS & 1.2354 & 0.7189 & 1.0603 \\
\hline & ML & 1.1378 & 0.7908 & 0.8905 \\
\hline & REML & 0.9858 & 0.6414 & 0.8523 \\
\hline & FFTW & 1.0750 & 0.7355 & 0.8424 \\
\hline
\end{tabular}

Table 6: Summary statistics of the parameter estimates, for a $\log \mathcal{N}$-distributed process $(N=10 \times 10)$, obtained with the different estimation methods.

\begin{tabular}{|c|c|c|c|c|c|c|}
\hline & $c_{0}$ & $\sigma^{2}$ & $a$ & $\nu$ & ASE & DASE \\
\hline \hline WLS & 0.000 & 16.140 & 1.329 & 2.448 & 0.029 & 0.549 \\
ML & 0.253 & 2.909 & 1.943 & 0.667 & 0.228 & 0.452 \\
REML & 0.252 & 2.933 & 1.978 & 0.663 & 0.227 & 0.452 \\
FFTW & 0.000 & 23.050 & 1.785 & 2.275 & 0.028 & 0.680 \\
\hline
\end{tabular}

Table 7: Variogram parameter estimates and cross-validation measures for the wind-speed data. ASE: averaged squared prediction error. DASE: dimensionless averaged squared error.

the spectral density estimation are eliminated. It is not difficult to see that the FFTW estimator $\tilde{\theta}$ is a weakly consistent estimator of $\theta_{0}$, assuming that the 


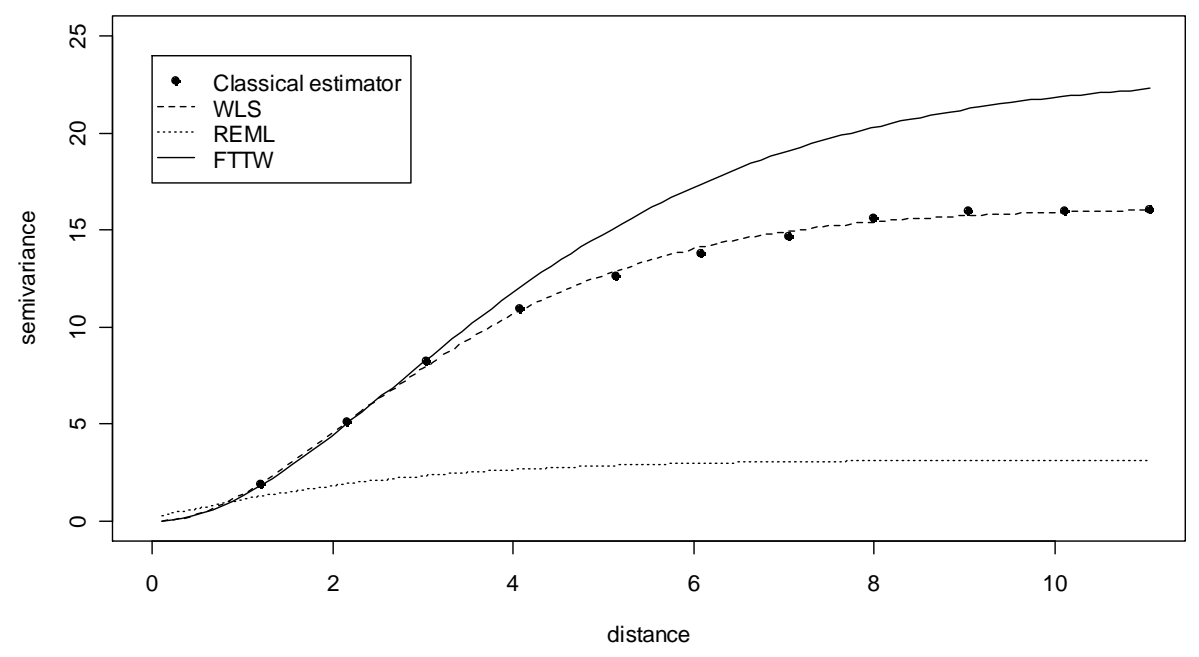

Figure 10: Variogram estimates for wind speed data. Points: classical estimator. Dashed line: WLS estimator. Dotted line: REML estimator (ML fit was almost identical). Solid line: FFTW estimator.

spectral density is bounded and bounded away from zero and it has first and second order bounded derivatives (asymptotically, the increasing and spacing parameters must satisfy that $n_{1}, n_{2} \rightarrow \infty, \Delta_{1}, \Delta_{2} \rightarrow 0$, and $\Delta_{l} n_{l} \rightarrow \infty$, for $l=1,2)$. In terms of strong convergence, this estimator has also a Gaussian limit, with limit variance as the estimator proposed by Guyon (1982). Nevertheless, the power of the new proposal is in its good performance for finite samples (as confirmed by the simulation study).

The comparisons made in the simulation study, reveal that the proposed method is competitive compared to the traditional estimation procedures in spatial statistics. In the simulations, FFTW clearly outperformed WLS estimation and had a similar or even better behaviour to ML procedures. It is important to point out that the FFTW estimation has a significantly lower computational cost than the ML methods, specially when the sample size is large (since FFT is used instead of covariance matrix factorization). For example, in the simulations with $N=15 \times 15$, the CPU time of the REML estimation 
was about 2.5 minutes whereas the FFTW method taked less than 20 seconds (on an AMD Athlon 64 3200+). Finally, this approximation can be applied in other cases, for instance, in spatio-temporal dependence modelling.

\section{Acknowledgments}

This work was partially supported by project MTM2005-00820 of the Spanish Ministry of Education and Science. In addition, research of Rubén FernándezCasal was partially supported by projects PGIDIT05TIC00701CT and PGIDIT07PXIB105259PR of the Xunta de Galicia.

\section{References}

Brillinger, D. R., 1981. Time series, 2nd Edition. Holden-Day Inc., Oakland, Calif., data analysis and theory, Holden-Day Series in Time Series Analysis.

Carroll, R. J., Ruppert, D., 1982. A comparison between maximum likelihood and generalized least squares in a heteroscedastic linear model. J. Amer. Statist. Assoc. 77 (380), 878-882.

Cressie, N., 1985. Fitting variogram models by weighted least squares. J. Internat. Assoc. Math. Geol. 17 (7), 563-586.

Cressie, N., Huang, H.-L., 1999. Classes of non-separable, spatio-temporal stationary covariance functions. Journal of the American Statistical Association $12,1330-1340$.

Cressie, N. A. C., 1993. Statistics for spatial data. Wiley Series in Probability and Mathematical Statistics: Applied Probability and Statistics. John Wiley \& Sons Inc., New York, a Wiley-Interscience Publication.

Crujeiras, R. M., Fernández-Casal, 2008. On the estimation of the spectral density for continuous spatial processes. Statistics To appear.

Crujeiras, R. M., Fernández-Casal, R., González-Manteiga, W., 2007. Comparing spatial dependence structures using spectral density estimators. Environmetrics 18, 793-808. 
Dahlhaus, R., Künsch, H., 1987. Edge effects and efficient parameter estimation for stationary random fields. Biometrika 74 (4), 877-882.

Fan, J., Zhang, W., 2004. Generalised likelihood ratio tests for spectral density. Biometrika 91 (1), 195-209.

Fernández-Casal, R., González-Manteiga, W., Febrero-Bande, M., 2003. Spacetime dependency modeling using general classes of flexible stationary variogram models. J. Geophys. Res. 108 (D24), 6-1 to 6-12.

Fuentes, M., 2002. Spectral methods for nonstationary spatial processes. Biometrika 89 (1), 197-210.

Goldberg, D. E., 1989. Genetic Algorithms in Search, Optimization, and Machine Learning. Addison-Wesley.

Guyon, X., 1982. Parameter estimation for a stationary process on a $d$ dimensional lattice. Biometrika 69 (1), 95-105.

Journel, A., Huijbregts, C., 1978. Mining Geostatistics. Academic Press, London.

Lahiri, S., Lee, Y., Cressie, N., 2002. On asymptotic distribution and asymptotic efficiency of least squares estimators of spatial variogram parameters. Journal of Statistical Planning and Inference 103, 65-85.

Mardia, K. V., Watkins, A., 1989. On multimodality of the likelihood in the spatial linear model. Biometrika 76, 289-295.

Priestley, M. B., 1981. Spectral analysis and time series. Vol. 1. Academic Press Inc. [Harcourt Brace Jovanovich Publishers], London, univariate series, Probability and Mathematical Statistics.

Ripley, B. D., 1988. Statistical inference for spatial processes. Cambridge University Press, Cambridge.

Robinson, P., 2007. Nonparametric spectrum estimation for spatial data. Journal of Statistical Planning and Inference. 137 (3), 1024-1034.

Robinson, P., Vidal Sanz, J., 2006. Modified whittle estimation of multilateral models on a lattice. J. Multivariate Anal. 97, 1090-1120. 
Stein, M. L., 1988. Asymptotically efficient prediction of a random field with a misspecified covariance function. Annals of Statistics 16, 55-63.

Stein, M. L., 1999. Interpolation of spatial data. Springer Series in Statistics. Springer-Verlag, New York, some theory for Kriging.

Whittle, P., 1954. On stationary processes in the plane. Biometrika 41, 434-449.

Yaglom, A. M., 1987. Correlation theory of stationary and related random functions. Vol. II. Springer Series in Statistics. Springer-Verlag, New York, supplementary notes and references.

Zimmerman, D., Zimmerman, M., 1991. A monte carlo comparison of spatial semivariogram estimators and corresponding ordinary kriging predictors. Technometrics 33, 77-91. 